\title{
Pathophysiology of Brain Injury and Neurological Outcome in Acute Respiratory Distress Syndrome: A Scoping Review of Preclinical to Clinical Studies
}

\author{
Merry Huang ${ }^{1}$, Aron Gedansky ${ }^{1}$, Catherine E. Hassett ${ }^{2}$, Carrie Price ${ }^{3}$, Tracey H. Fan ${ }^{1}$, R. Scott Stephens ${ }^{4}$, \\ Paul Nyquist ${ }^{5}$, Ken Uchino ${ }^{2}$ and Sung-Min $\mathrm{Cho}^{5^{*}}$ (D)
}

๑ 2021 Springer Science+Business Media, LLC, part of Springer Nature and Neurocritical Care Society

\begin{abstract}
Acute respiratory distress syndrome (ARDS) has been associated with secondary acute brain injury (ABI). However, there is sparse literature on the mechanism of lung-mediated brain injury and prevalence of ARDS-associated secondary ABI. We aimed to review and elucidate potential mechanisms of ARDS-mediated ABI from preclinical models and assess the prevalence of ABI and neurological outcome in ARDS with clinical studies. We conducted a systematic search of PubMed and five other databases reporting ABI and ARDS through July 6,2020 and included studies with $A B I$ and neurological outcome occurring after ARDS. We found 38 studies (10 preclinical studies with 143 animals; 28 clinical studies with 1175 patients) encompassing 9 animal studies $(n=143), 1$ in vitro study, 12 studies on neurocognitive outcomes ( $n=797)$, 2 clinical observational studies $(n=126), 1$ neuroimaging study $(n=15)$, and 13 clinical case series/reports $(n=15)$. Six ARDS animal studies demonstrated evidence of neuroinflammation and neuronal damage within the hippocampus. Five animal studies demonstrated altered cerebral blood flow and increased intracranial pressure with the use of lung-protective mechanical ventilation. High frequency of ARDS-associated secondary $\mathrm{ABI}$ or poor neurological outcome was observed ranging $82-86 \%$ in clinical observational studies. Of the clinically reported ABIs (median age 49 years, $46 \%$ men), the most common injury was hemorrhagic stroke (25\%), followed by hypoxic ischemic brain injury (22\%), diffuse cerebral edema (11\%), and ischemic stroke (8\%). Cognitive impairment in patients with ARDS $(n=797)$ was observed in $87 \%$ (range $73-100 \%)$ at discharge, $36 \%$ (range $32-37 \%$ ) at 6 months, and 30\% (range 25-45\%) at 1 year. Mechanisms of ARDS-associated secondary ABI include primary hypoxic ischemic injury from hypoxic respiratory failure, secondary injury, such as lung injury induced neuroinflammation, and increased intracranial pressure from ARDS lung-protective mechanical ventilation strategy. In summary, paucity of clinical data exists on the prevalence of ABI in patients with ARDS. Hemorrhagic stroke and hypoxic ischemic brain injury were commonly observed. Persistent cognitive impairment was highly prevalent in patients with ARDS.
\end{abstract}

Keywords: Acute respiratory distress syndrome, Acute brain injury, Neurological outcome, Lung-brain interaction

\footnotetext{
*Correspondence: csungmi1@jhmi.edu

${ }^{5}$ Division of Neuroscience Critical Care, Departments of Neurology, Neurosurgery, Anesthesiology and Critical Care Medicine, Johns Hopkins University, 600 N. Wolfe Street, Phipps 455, Baltimore, MD, USA

Full list of author information is available at the end of the article
}

This article is related to the Invited Commentary available at https://link. springer.com/article/10.1007/s12028-021-01310-4.

\section{Springer}

\section{Introduction}

Acute respiratory distress syndrome (ARDS) is characterized by diffuse inflammation and noncardiogenic pulmonary edema due to increased alveolar-capillary vascular permeability, which leads to severe hypoxemia and respiratory failure requiring mechanical 
ventilatory support [1]. ARDS has a prevalence of 19 to $23 \%[2,3]$ of all patients who are mechanically ventilated and carries a significant hospital mortality rate of 35 to $48 \%$ [2, 4, 5]. Survivors of ARDS have long-term morbidity, including ventilatory deficits, decreased physical function, and neuropsychiatric morbidities [1, 6-8].

Acute brain injury (ABI) can lead to development of ARDS. For instance, approximately one in four patients with isolated traumatic brain injury or spontaneous intracranial hemorrhage developed ARDS during hospitalization $[9,10]$. On the other hand, ARDS can lead to $\mathrm{ABI}$ and thus impair neurological function. These observations suggest the existence of cross talk between the lung and the brain [11]. Currently, the mechanism of lung-mediated brain injury is not well understood. Given that lung injury may lead to profound hypoxia, such as in ARDS, it is plausible that highly metabolic organs, such as the brain, may be affected [12]. Further, lung injury leads to release of systemic inflammatory mediators that can cross the blood-brain barrier and cause cerebral dysfunction [13]. The use of mechanical ventilation in lung injury may also alter serum carbon dioxide concentrations, which, in turn, can affect cerebral hemodynamics [14].

To date, there has not been a comprehensive effort to review $\mathrm{ABI}$ and neurological outcome in patients with ARDS. Furthermore, sparse evidence exists on the mechanisms of ARDS-associated secondary ABI. Herein, we aimed to (1) review preclinical studies of ARDS-associated secondary ABI to understand the pathophysiology of ARDS-mediated brain injury and (2) review clinical studies of ARDS-associated secondary ABIs and neurological outcome to understand the prevalence of $\mathrm{ABI}$ in patients with ARDS.

\section{Methods}

\section{Search Strategy}

This scoping review was reported following the framework described in Arksey and O'Malley [15] and according to the Preferred Reporting Items for Systematic Reviews and Meta-Analyses guidelines [16]. We searched PubMed, both legacy and new, via NCBI, Embase via Elsevier, the Cochrane Library via Wiley, Web of Science Core Collection via Clarivate, and Scopus via Elsevier. The search included controlled vocabulary subject headings and keywords related to ARDS and ABI from inception to July 6, 2020. An effort was made to account for plurals, acronyms, and synonyms. The detailed search strategy is available in Appendix 1 . The results were deduplicated and uploaded to Covidence.

\section{Inclusion Criteria}

Inclusion criteria were applied following the population, intervention, comparator, outcome, and study design approach [16]. We included (1) randomized controlled trials (RCTs), observational studies, case series/reports, and animal and in vitro studies; (2) adult patients ( $>18$ years); (3) ARDS defined by the Berlin Criteria or American-European Consensus Conference Criteria [17, 18]; and (4) studies with ABI occurring after ARDS. The search was not limited to English language, and non-English articles were translated and reviewed for inclusion eligibility.

\section{Exclusion Criteria}

We excluded (1) editorials, commentaries, and reviews; (2) studies with ABI occurring after implementation of ECMO for ARDS because of an increased risk of ABI in this patient cohort [19]; (3) studies with acute lung injury that did not meet ARDS criteria; and (4) studies with ABI occurring prior to ARDS.

\section{Study Selection and Data Extraction}

Two reviewers ( $M H, A G)$ independently assessed the literature results for eligibility. A third reviewer $(\mathrm{CH}$ or $\mathrm{SM}-\mathrm{C}$ ) resolved any disagreements on inclusion/exclusion of the literature. Covidence, Cochrane's online systematic review platform, was used to streamline the review process. Articles meeting inclusion criteria were retrieved and the full text was reviewed. References of included studies were screened and included based on inclusion and exclusion criteria. Data from eligible articles were extracted and recorded in an excel spreadsheet (Microsoft, Redmond, WA). Extracted data included authors, title of article, date of publication, name of journal, type of article, objectives, methods, key findings, brain injury, neurological outcome, sample size, demographics, hospital length of stay, ARDS severity, $\mathrm{PaO}_{2}$ to $\mathrm{FiO}_{2}$ ratio, Acute Physiology and Chronic Health Evaluation II (APACHE II) score, and other quantitative results, if applicable.

\section{Primary Outcomes}

Primary outcomes were mechanisms of ARDS-mediated secondary ABI. Clinical outcomes were $\mathrm{ABI}$ and neuroimaging abnormalities reported during index hospitalization and neurological outcome at discharge and follow-up. ABI included hypoxic ischemic brain injury (HIBI), ischemic stroke, and hemorrhagic stroke. Neuroimaging abnormalities included brain atrophy and cerebral microbleed (CMB). Neurological outcomes included cognitive function and Cerebral 
Performance Category score (defined as favorable neurological outcome if $<3$ ).

\section{Quality Assessment and Risk of Bias}

The Cochrane Risk of Bias assessment tool was used to assess risk of bias in RCT in eight domains [20]. The trial was considered high risk if at least one domain was rated as a high risk and low risk if all domains were judged as low. The Newcastle-Ottawa Scale (NOS) was used to evaluate the risk of bias of cohort studies. The NOS scores assigned points to three domains: patient selection, comparability, and assessment of outcome or exposure [21]. Studies scoring 6 or more points were considered to have a low risk for bias. The Murad tool was used to assess the quality of case reports/series [22]. The study was considered to be high quality if it had adequate data in selection, ascertainment, causality, and reporting. Publication quality was assessed independently by two investigators (MH, AG). Any discrepancies were resolved in consensus with a third investigator $(\mathrm{CH})$.

\section{Statistical Analysis}

Data on age from all studies were collected and reported as an overall median. The portion of cognitive impairment was collected across all cognitive studies and reported as a median and range. The prevalence of ABI was calculated based on the number of patients with $A B I$ divided by the total number of patients with ARDS. Meta-analysis of these studies was not performed because of limited number of studies and high heterogeneity of the included studies.

\section{Results}

The search yielded 31,327 citations. After 17,414 duplicates were removed, 13,913 citations remained. Following abstract screening, 382 articles were eligible for full text review. Of these studies, 344 were excluded based on exclusion criteria, including 3 studies with overlapping patient data, leaving the final 38 studies (10 preclinical studies with 143 animals; 28 clinical studies with 1032 patients) for the systematic review (Fig. 1), including 9 animal studies ( $n=143), 1$ in vitro study, 12 studies on neurological cognitive outcomes $(n=797), 2$ clinical observational studies $(n=126), 1$ neuroimaging study $(n=15)$, and 13 clinical case series/reports $(n=15)$. A summary of the included studies is described in Supplemental Table 1.

\section{Preclinical Animal and In Vitro Studies}

Of the ten preclinical studies, nine studies $(n=143)$ involved porcine with ARDS and one study was in vitro. ARDS was induced by one of three methods: (1) injection of oleic acid directly causing lung injury, (2) repeated pulmonary lavage causing surfactant depletion and poor gas exchange, or (3) hydrochloric acid aspiration directly causing lung injury.

Six animal studies $(n=100)$ demonstrated neuroinflammation after ARDS with increased serum inflammatory markers, including interleukin- 6 and tumor necrosis factor $\alpha$, which correlated with elevated markers of neuronal damage, including neuron-specific enolase and S100 calcium-binding protein B [23-28]. Two studies $(n=12)$ directly showed neuronal damage and perivascular inflammation in the pyramidal layer of the hippocampal region on both light microscopy and histopathological analysis in ARDS animals [24, 29].

Five studies $(n=59)$ described adverse effects of mechanical ventilation in ARDS, including increased intracranial pressure (ICP), neuroinflammation, and disruption of cerebral oxygen metabolism [23, 26, 30, $31]$. In one study, elevated plateau pressure was most strongly correlated with increased ICP when monitored with intraparenchymal Camino ICP monitoring device, followed by increased $\mathrm{PaCO}_{2}$ and increased central venous pressure (CVP) [32]. Concomitant intraabdominal pressure through induction of pneumoperitoneum in lung injury was associated with further increases in ICP secondary to increased plateau pressure and CVP [32]. One in vitro study demonstrated neuronal secretion of inflammatory markers and apoptosis, in which these cells were mixed with the medium from lipopolysaccharide-induced injured airway epithelial cells [27]. Potential mechanisms of brain injury in ARDS in preclinical models are summarized in Table 1 and graphically represented in Fig. 2.

\section{Clinical Studies on Brain Injury After ARDS Observational Studies}

There was a paucity of literature on ARDS-associated secondary brain injury, with only two observational studies $(n=126)$. Among these studies, the median age was 61 year (63\% men) and 53\% of the patients had sepsis or pneumonia. Severe ARDS was most common (43\%), followed by moderate (40\%) and mild ARDS (17\%). High frequency of ABI or poor neurological outcome after ARDS was observed ranging 82-86\%. Because of the heterogeneity and scarcity of reported outcomes, a cumulative statistical analysis on neurological outcome involving these two clinical studies was not preformed. One autopsy study $(n=7)$ found HIBI to be the most common ABI (86\%) after ARDS [33]. Another study found poor neurological outcome at 28 days was more frequently seen in patients with cardiac arrest with ARDS (82\%), compared with those without ARDS (61\%) [34]. 


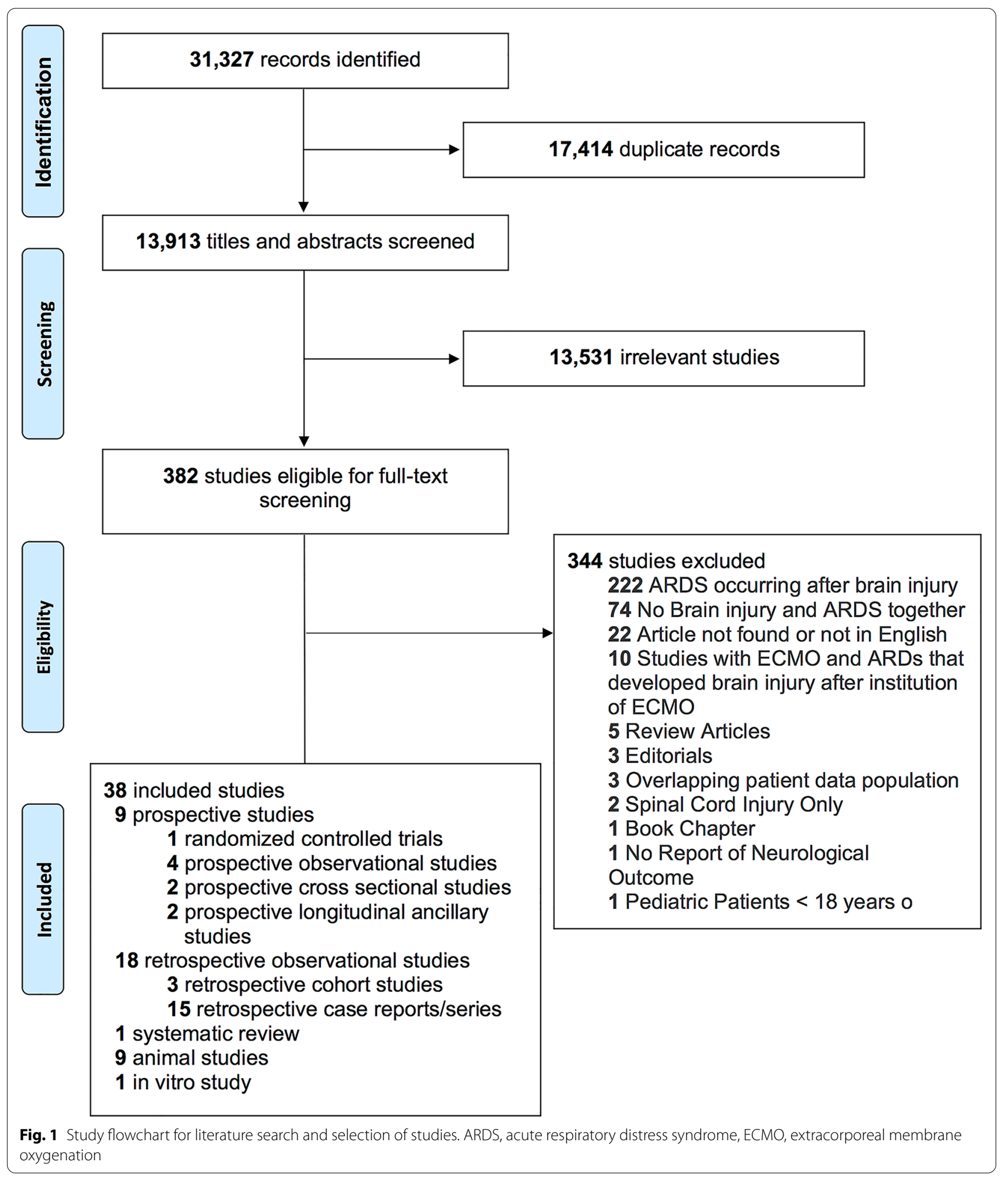


Table 1 Potential mechanisms of brain injury in acute respiratory distress syndrome in preclinical models

\begin{tabular}{|c|c|c|c|c|c|}
\hline \multicolumn{6}{|l|}{ Neuroinflammation } \\
\hline Study & Animal & $n$ & Age, sex, weight & $\begin{array}{l}\text { Lung } \\
\text { injury } \\
\text { method }\end{array}$ & Result \\
\hline Bickenbach et al. 2011 [24] & Porcine & 10 & Female sex, $31.8 \pm 1.2 \mathrm{~kg}$ & RBL & $\begin{array}{l}\text { Increased serum IL-6 } \\
\text { Inflammation on brain pathology }\end{array}$ \\
\hline Fries et al. 2005 [29] & Porcine & 14 & Female sex, $29 \pm 2 \mathrm{~kg}$ & RBL & $\begin{array}{l}\text { Increased serum S100B } \\
\text { Shrunken neurons of the pyramidal cell layer in the } \\
\text { hippocampal CA1 on pathology }\end{array}$ \\
\hline Heuer et al. 2011 [25] & Porcine & 28 & Female sex, 52-65 kg & OAl & $\begin{array}{l}\text { Increased serum NSE } \\
\text { Damage to the hippocampus and cerebral edema } \\
\text { on pathology }\end{array}$ \\
\hline Kamuf et al. 2017 [28] & Porcine & 32 & NA & OAl & $\begin{array}{l}\text { Decreased in IL-6 expression in the brain with treat- } \\
\text { ment of antiinflammatory agent }\end{array}$ \\
\hline Rodriguez-Gonzalez et al. 2015 [27] & In vitro & NA & LPS induced alveolar cell injury & NA & $\begin{array}{l}\text { Increased cellular secretion of S100B, NSE, IL-6 } \\
\text { Neuronal necrosis and apoptosis }\end{array}$ \\
\hline \multicolumn{6}{|l|}{$\begin{array}{l}\text { Adverse effects of lung-protective } \\
\text { mechanical ventilatory strategy }\end{array}$} \\
\hline Bickenbach et al. 2009 [23] & Porcine & 10 & Female sex, $30.2 \pm 2.0 \mathrm{~kg}$ & RBL & $\begin{array}{l}\text { HT ventilation leads to increased serum S100B, IL-6, } \\
\text { venous } \mathrm{O} 2 \text { concentration, and cerebral lactate } \\
\text { levels }\end{array}$ \\
\hline Kamuf et al. 2018 [26] & Porcine & 20 & Male sex, 24-31 kg & $\mathrm{OAI} / \mathrm{RBL}$ & $\begin{array}{l}\text { MV results in increased IL-6 and TNFa expression in } \\
\text { hippocampus }\end{array}$ \\
\hline Klein et al. 2013 [30] & Porcine & 12 & Juvenile, $25-27$ kg & RBL & $\begin{array}{l}\text { MV induced cyclic oscillations in peripheral } \mathrm{PaO} 2 \text { are } \\
\text { transmitted to cerebral } \mathrm{PaO} 2\end{array}$ \\
\hline Kreyer et al. 2013 [31] & Porcine & 9 & $38.2 \pm 5.3 \mathrm{~kg}$ & HA & $\begin{array}{l}\text { LT ventilation results in hypercapnia and increased } \\
\text { regional CBF }\end{array}$ \\
\hline Zampieri et al. 2011 [32] & Porcine & 8 & Female sex, 35-42 kg & $\mathrm{RBL}$ & $\begin{array}{l}\text { Plateau airway pressure, CO2 arterial pressure, and } \\
\text { CVP are associated with increased ICP }\end{array}$ \\
\hline
\end{tabular}

CA1, cornu ammonis, CBF, cerebral blood flow, CVP, central venous pressure, HA, hydrochloric acid aspiration, $\mathrm{HT}$, high tidal, ICP, intracranial pressure, IL-6, interleukin-6, LT, low tidal, LPS, lipopolysaccharide, MV, mechanical ventilation, NA, not available, NSE, neuron specific enolase, OAI, oleic acid injection, PaO2, partial pressure of oxygen, pECLA, percutaneous extracorporeal lung assist, RBL, repetitive bronchoalveolar lavage, R/D, recruitment/derecruitment, S100B, calcium-binding protein B, S100 calcium binding protein, TNF, tumor necrosis factor

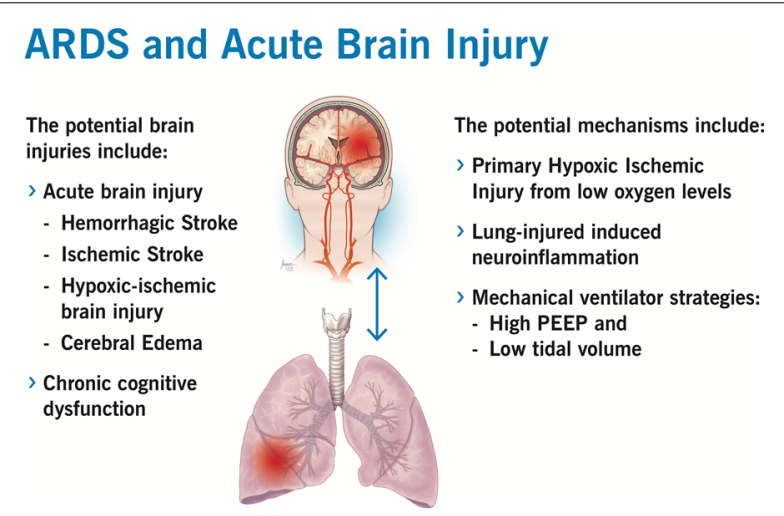

Fig. 2 ARDS-associated secondary acute brain injury and potential mechanism ARDS-mediated brain injury. ARDS, acute respiratory distress syndrome, PEEP, positive end-expiratory pressure

\section{Case Reports/Case Series}

Among case reports/series (13 studies, $n=15)$, the median age was 49 years (33\% men) and $87 \%(n=13)$ suffered from sepsis. Of these patients who were septic, $77 \%(n=10)$ had ARDS secondary to respiratory viral illness, including seven $\mathrm{H} 1 \mathrm{~N} 1$, two SARS-CoV-2, and one MERS-CoV. Among the studies that reported ARDS severity $(n=4)$, severe ARDS was the most common (75\%), and 57\% died from ARDS and its complications. The most common reported ABI was hemorrhagic stroke $(40 \%)$, followed by diffuse cerebral edema (20\%), HIBI (13\%), and ischemic stroke (13\%). Of the patients with hemorrhagic stroke, 33\% had CMBs. Other ABIs included posterior reversible encephalopathy syndrome and delayed posthypoxic leukoencephalopathy. A summary of reported clinical brain injuries in all case reports and clinical studies of ARDS is described in Table 2 and graphically represented in Fig. 2.

\section{Neuroimaging Studies}

There was only one neuroimaging study $(n=15)$ involving head computed tomography of patients with ARDS, with a median age of 39.2 years ( $60 \%$ men). Severity and etiology of ARDS were not reported. Brain atrophy was 
Table 2 Types of reported acute brain injuries in ARDS

\begin{tabular}{|c|c|}
\hline Characteristics & $\begin{array}{l}\text { Patients } \\
\text { with ARDS } \\
\text { and ABI }(n=30)\end{array}$ \\
\hline \multicolumn{2}{|l|}{ Demographics } \\
\hline Age, median (IQR) & $50(44-54)$ \\
\hline Male sex & $8(30 \%)$ \\
\hline \multicolumn{2}{|l|}{ Past medical history } \\
\hline Lung disease & $8(33.3 \%)$ \\
\hline Malignancy & $3(12.5 \%)$ \\
\hline Obesity & $3(12.5 \%)$ \\
\hline Smoking & $2(8.3 \%)$ \\
\hline Hypertension & $1(4.2 \%)$ \\
\hline Atrial fibrillation & $1(4.2 \%)$ \\
\hline Hypothyroidism & $1(4.2 \%)$ \\
\hline Alcohol use & $1(4.2 \%)$ \\
\hline Cardiac disease & $1(4.2 \%)$ \\
\hline Diabetes & $1(4.2 \%)$ \\
\hline Other & $2(8.3 \%)$ \\
\hline \multicolumn{2}{|l|}{ Etiology of ARDS } \\
\hline Sepsis or pneumonia & $19(95 \%)$ \\
\hline Drug reaction & $1(5 \%)$ \\
\hline \multicolumn{2}{|l|}{ ARDS variables } \\
\hline Mild ARDS & $1(8.3 \%)$ \\
\hline Moderate ARDS & $0(0 \%)$ \\
\hline Severe ARDS & $11(91.7 \%)$ \\
\hline \multicolumn{2}{|l|}{ Types of ABI } \\
\hline Brain atrophy & $9(25 \%)$ \\
\hline Hypoxic ischemic brain injury & $8(22.2 \%)$ \\
\hline Subarachnoid hemorrhage & $5(13.9 \%)$ \\
\hline Cerebral edema & $4(11.1 \%)$ \\
\hline Ischemic stroke & $3(8.3 \%)$ \\
\hline Intracranial hemorrhage & $2(5.6 \%)$ \\
\hline Critical-illness associated microbleeds & $2(5.6 \%)$ \\
\hline Delayed posthypoxic leukoencephalopathy & $2(5.6 \%)$ \\
\hline Posterior reversible leukoencephalopathy & $1(2.8 \%)$ \\
\hline Survival & $14 / 30(47 \%)$ \\
\hline
\end{tabular}

Male gender, past medical history, etiology of ARDS, and ARDS variables were not reported in all studies; percentages are of all patients in all studies which reported these variables

$\mathrm{ABI}$, acute brain injury, ARDS, acute respiratory distress syndrome, IQR, interquartile range

the most common finding on head computed tomography (53\%) with prominent involvement of the hippocampus (71\%) [35].

\section{Clinical Studies on Neurological and Cognitive Outcomes}

The observational studies on cognitive outcome in ARDS included 797 patients (median age: 46 years, $45 \%$ men). The most common cognitive assessments used were Wechsler Adult Intelligence Scale-Third Edition and
Weschler Memory Scale-Third Edition. A full list of cognitive assessment tools used in these studies is listed in Supplemental Table 2. The median hospital stay in all patients was 38.9 days, median intensive care unit (ICU) stay was 14.6 days, and median duration of intubation was 19.7 days. Median $\mathrm{PaO}_{2}$ to $\mathrm{FiO}_{2}$ ratio at admission was 154.7 and median APACHE II score at admission was 21 (Supplemental Table 3). The median proportion of cognitive impairment across all studies was $87 \%$ (range $73-100 \%$ ) at discharge, $36 \%$ (range $32-37 \%$ ) at 6 months, and $30 \%$ (range $25-45 \%$ ) at 1 year (Table 3 ). Among the studies, cognitive impairment was not found to be associated with age, sex, APACHE II, hospital or ICU duration, mechanical ventilation duration, or use of sedatives, narcotics, and paralytics. Two studies found cognitive impairment was associated with hypotension (mean arterial pressure $<50 \mathrm{~mm} \mathrm{Hg}$ ) during ICU stay and low $\mathrm{PaO}_{2}$ on admission $(71 \mathrm{~mm} \mathrm{Hg})[36,37]$. One study found low CVP and conservative fluid management in ARDS were associated with cognitive dysfunction, possibly due to decreased cerebral perfusion [37]. Another study found hyperglycemia (highest glucose levels $>153.5 \mathrm{mg} / \mathrm{dL}$ ) during ICU stay was associated with cognitive impairment [38]. A summary of associated factors in the development of cognitive impairment following ARDS is described in Supplemental Table 4.

\section{Risk of Bias Assessment}

The Cochrane tool showed high risk of bias for three animal RCTs because of inadequate information regarding randomization. The remainder of RCTs were low risk (Supplemental Table 5). The NOS was conducted on two cohort studies and did not indicate high risk of bias for any study with median NOS score of 6.5 (Supplemental Table 6). All included case series had adequate data on case selection, exposure and outcome ascertainment, and causality, with a median score of 6 .

\section{Discussion}

We performed a scoping review on both preclinical and clinical studies reporting ABI and neurological outcome in ARDS to better understand the pathophysiology behind ARDS-mediated secondary brain injury and to assess the prevalence of clinical ARDS-associated secondary. Our review found evidence of neuroinflammation and altered cerebral hemodynamics with use of mechanical ventilation in preclinical animal studies with ARDS. There was an overall paucity of clinical reports on ARDS-associated secondary ABIs. However, we found a high frequency of $A B I$ based on few observational studies. Types of reported ABI in patients with ARDS were hemorrhagic stroke including intracerebral hemorrhage, subarachnoid hemorrhage, and $\mathrm{CMB}$, ischemic injury 
Table 3 Studies on cognitive impairment in acute respiratory distress syndrome

\begin{tabular}{|c|c|c|c|c|c|}
\hline Study & Type of study & $n$ & Age & Male sex & $\begin{array}{l}\text { Cognitive } \\
\text { impair- } \\
\text { ment }\end{array}$ \\
\hline \multicolumn{6}{|c|}{ Cognitive impairment at discharge } \\
\hline Hopkins et al. 1999 [36] & Prospective observational study & 55 & 46 & 25 & $100 \%$ \\
\hline \multirow[t]{2}{*}{ Hopkins et al. 2004 [64] } & Prospective observational study & 74 & 46 & 33 & $73 \%$ \\
\hline & & & Median (IQR): & $87 \%(73-100 \%)$ & \\
\hline \multicolumn{6}{|c|}{ Cognitive impairment at 6 months } \\
\hline Jackson et al. 2003 [67] & Prospective observational study & 34 & 53 & 18 & $32 \%^{\mathrm{a}}$ \\
\hline Needham et al. 2013 & Prospective observational study & 174 & 47 & 87 & $36 \%$ \\
\hline \multirow[t]{2}{*}{ Needham et al. 2016} & Randomized controlled trial & 189 & 50 & 89 & $37 \%$ \\
\hline & & & Median (IQR): & $36 \%(32-37 \%)$ & \\
\hline \multicolumn{6}{|c|}{ Cognitive impairment at 1 year } \\
\hline Hopkins et al. 1999 [36] & Prospective observational study & 55 & 46 & 25 & $30 \%$ \\
\hline Hopkins et al. 2004 [64] & Prospective observational study & 74 & 46 & 33 & $46 \%$ \\
\hline Mikkelsen et al. 2012 [37] & Prospective observational study & 75 & 50 & 32 & $55 \%$ \\
\hline Needham et al. 2013 & Prospective observational study & 174 & 47 & 87 & $25 \%$ \\
\hline \multirow[t]{2}{*}{ Needham et al. 2016} & Randomized controlled trial & 189 & 50 & 89 & $29 \%$ \\
\hline & & & Median (IQR): & $30 \%(25-45 \%)$ & \\
\hline \multicolumn{6}{|c|}{ Cognitive impairment at 2 years } \\
\hline Adhikari et al. 2009 & Prospective observational study & 71 & 42 & 38 & $8-20 \%^{b}$ \\
\hline Mikkelsen et al. 2009 & Prospective cross-sectional study & 79 & 43 & 12 & $56 \%$ \\
\hline \multicolumn{6}{|c|}{ Cognitive impairment at 6 years } \\
\hline Rothenhäusler et al. 2001 & Retrospective study & 46 & $N A^{c}$ & 24 & $24 \%$ \\
\hline
\end{tabular}

including ischemic stroke and HIBI, diffuse and focal hippocampal brain atrophy, and diffuse cerebral edema. Less commonly reported ABIs were posterior reversible encephalopathy syndrome and delayed posthypoxic leukoencephalopathy.

Our study suggests, based on preclinical studies, that ARDS induced ABI is mediated through two major mechanisms: (1) neuroinflammation and (2) neurological adverse effects of lung-protective mechanical ventilation strategy. First, direct alveolar stretching and lung injury have been shown to promote release of proinflammatory cytokines, including TNF-alpha, IL-1beta, and IL-6 $[11,24-26,39,40]$. Such inflammation can directly cause neuronal apoptosis [41]. Interestingly, the hippocampal region has a high density of IL-1 receptors, which may explain the common occurrence of hippocampal injury seen in patients with ARDS given its vulnerability to inflammation as well as hypoxic insult [42]. Second, although ARDS mechanical ventilatory strategies have demonstrated to improve survival, they may contribute or worsen brain injury in some patients $[14,43$, 44]. Lung-protective strategy with low tidal ventilation to reduce lung strain is associated with hypercapnia and subsequent cerebral vasodilation, increased cerebral blood flow, and increased ICP, which may further worsen $\mathrm{ABI}$ and increase risk for cerebral ischemia [14]. High positive end-expiratory pressure (PEEP) is another ventilation strategy used in ARDS to improve oxygenation, prevent alveolar collapse, and reduce atelectrauma [45]. Theoretically, high PEEP may cause increased ICP through multiple mechanisms, including (1) increased cerebral vasodilation from elevated intrathoracic pressure and subsequent decrease in mean arterial pressure (2) decreased cerebral venous outflow from increased CVP, and (3) reduced cerebrospinal fluid outflow from increased spinal pressure [46]. However, previous animal and human studies did not reveal that PEEP had such effect an on ICP although these studies did not involve subjects with ARDS who may have decreased lung compliance and therefore be more sensitive to intrathoracic transmission of PEEP $[46,47]$. One ARDS animal study found plateau pressure, CVP, and $\mathrm{PaCO}_{2}$ were associated with increased ICP [32]. Further, ARDS animals with concomitant increased intraabdominal pressure 
had greater increases in ICP compared with lung injured animals alone, likely due to greater elevation in plateau pressure. A recent clinical study also found that high lung compliance is associated with favorable neurological outcome in patients with ARDS [48]. It is therefore plausible that select patients with decreased lung compliance such as in ARDS may be more sensitive to effects of PEEP on ICP, particularly when combined with low tidal ventilation leading to permissive hypercapnia and subsequent cerebral vasodilation. However, further clinical research on the effects of PEEP on ICP in patients with elevated plateau pressure is needed.

Our study demonstrated that the most common clinical ABI after ARDS was hemorrhagic stroke (25\%). The mechanism of hemorrhagic stroke in patients with ARDS is not entirely clear given limited reporting. One potential mechanism can be elucidated from occurrence of CMBs in patients with ARDS. Two ARDS case reports showed diffuse CMBs predominantly involving the cerebellum, brainstem, and juxtacortical white matter with sparing of the cortex, which may be consistent with Critical Illness Associated Microbleeds [49, 50]. These CMBs are thought to be due to hypoxemia and inflammation causing endothelial dysfunction, breakdown of bloodbrain barrier, and subsequent extravasation of erythrocytes [51]. ARDS is characterized by hypoxemia and an increased systemic inflammatory state, which can result in endothelial dysfunction and therefore contribute to CMBs, a phenotype of cerebral small vessel disease [5153], which may further evolve to hemorrhagic stroke during acute illness.

Ischemic brain injury was also commonly observed in ARDS. The mechanisms of ischemic stroke included watershed infarcts in the setting of septic shock and gas emboli secondary to barotrauma from mechanical ventilation [33, 54]. An autopsy study on patients with ARDS showed that HIBI was most commonly observed in hippocampus, specifically the pyramidal neurons in the CA1 region [33]. The hippocampus is known to be selectively vulnerable to ischemic injury due to high metabolic demand [55, 56]. Possible mechanisms of HIBI include prolonged hypoxemia from impaired exchange, particularly those with severe-refractory ARDS, and hypotension secondary to concomitant sepsis and shock physiology in ARDS. These factors disrupt oxygen and glucose delivery to brain tissue, leading to mitochondrial dysfunction and failure of energy-dependent ion chains subsequently causing neuronal apoptosis, necrosis, and cytotoxic edema [57]. Several cases of cerebral edema were reported in patients with ARDS, likely resulting from consequences of HIBI [58-60]. Diffuse cerebral atrophy was also commonly reported in patients with ARDS [35] although it is uncertain if this was caused by acute inflammation or hypoxemia from ARDS due to the absence of pre-ARDS imaging studies as a control. However, previous studies have described cerebral atrophy in patients with chronic hypoxemia, such as in obstructive sleep apnea and chronic obstructive pulmonary disease $[61,62]$. Additionally, brain atrophy has been observed in patients following cardiac arrest [63]. Further research is needed to assess the severity of ARDS and its impact on ischemic ABI.

It is important to highlight that survivors of ARDS commonly suffer from short-term and long-term cognitive impairment including memory, attention, and concentration domains [36]. Hypotension and hypoxemia were important risk factors associated with cognitive impairment [37, 64]. Decreased perfusion and refractory hypoxia may injure areas vulnerable to ischemic insult including hypothalamus, thalamus, and areas of the cortex, all of which are closely involved in neurocognitive function [63]. We found that cognitive function in ARDS survivors did not improve at 1-2 years after ARDS [65] and patients who did not recall their ICU stay or had fewer years of education (14.1 vs. 11.3 years) had greater degree of cognitive dysfunction [64, 66, 67]. Despite the common occurrence of long-term cognitive impairment in ARDS survivors, sparse data exist on preventative and therapeutic interventions to mitigate poor cognitive outcome in ARDS.

Our study has several limitations. First, the paucity of available studies and high degree of heterogeneity across both clinical and preclinical studies limit our ability to adequately assess the prevalence of ARDS-associated ABI. Further, it is possible that the prevalence of ABI may have been overestimated given that the included studies assessed more patients who were critically ill. Conversely, $A B I$ may be underestimated given that it can be underrecognized by clinicians $[2,68]$. As the severity of ARDS was not consistently reported, the impact of ARDS severity on ABI and neurological outcome could not be accurately assessed. Also, included studies did not report some important factors such as stroke risk factors that may have confounded the reported ABIs. A recent systematic review demonstrated that delirium and cognitive impairment were associated with mechanical ventilation, and therefore, it is possible that poor neurological outcome may be attributed to mechanical ventilation alone [69]. However, that study was not focused on subjects with ARDS and our study only included animals and patients with ARDS and provides a comprehensive review of ABI after ARDS. Second, cognitive assessment tools varied among different studies with significant heterogeneity and the interpretation of the data should be done with caution. These ARDS cognitive studies also lacked control group without baseline assessment prior 
to critical illness. Additionally, no studies accounted for ABIs in the development of cognitive impairment. Third, many case reports/series of ARDS included upper respiratory viral infection and sepsis leading to additional medical problems, which may have also contributed to ABIs. However, the animal studies without sepsis have robustly demonstrated the evidence for neuroinflammation in ARDS. Lastly, there were three animal RCTs that were determined to have high risk bias due to unclear randomization process. We included these studies in our review given that randomization was used to determine type of lung injury method to generate ARDS animal model, which did not have any effect on our outcome in reviewing the pathophysiology of ARDS-mediated brain injury.

\section{Conclusions}

Preclinical studies suggest that mechanisms of ARDSassociated ABI include primary hypoxic ischemic injury from hypoxic respiratory failure and secondary injury from lung injury induced neuroinflammation and increased ICP from lung-protective mechanical ventilatory strategies.

Paucity of clinical data exists on prevalence of ABI in patients with ARDS. Hemorrhagic stroke, HIBI, and brain atrophy were most observed ABIs. ARDS survivors have a high prevalence of cognitive impairment that may persist at 2 years.

\section{Supplementary Information}

The online version contains supplementary material available at https://doi. org/10.1007/s12028-021-01309-x.

\section{Author details \\ ${ }^{1}$ Department of Neurology, Neurological Institute, Cleveland Clinic, Cleveland, $\mathrm{OH}$, USA. ${ }^{2}$ Cerebrovascular Center, Neurological Institute, Cleveland Clinic, Cleveland, OH, USA. ${ }^{3}$ Albert S. Cook Library, Towson University, Towson, MD, USA. ${ }^{4}$ Division of Pulmonary and Critical Care Medicine, Department of Medi- cine, Johns Hopkins University, Baltimore, MD, USA. ${ }^{5}$ Division of Neurosci- ence Critical Care, Departments of Neurology, Neurosurgery, Anesthesiology and Critical Care Medicine, Johns Hopkins University, 600 N. Wolfe Street, Phipps 455, Baltimore, MD, USA.}

\section{Author contributions}

Study concept and design: MH, SMC. Acquisition, analysis, or interpretation of data: MH, AG, CEH, THF. Statistical analysis: MH. Tables and figures: MH. First drafting of the article: MH, SMC. Critical revision for important intellectual content and final approval of the article: CMC, KU, RSS, PN. All authors approve of the final manuscript.

\section{Conflict of interest}

The authors declare that they have no conflicts of interest.

\section{Ethical approval}

All ethical guidelines and use of informed consent where applicable were followed.

\section{Publisher's Note}

Springer Nature remains neutral with regard to jurisdictional claims in published maps and institutional affiliations.
Received: 21 April 2021 Accepted: 21 June 2021

Published online: 23 July 2021

\section{References}

1. Matthay MA, Zemans RL, Zimmerman GA, et al. Acute respiratory distress syndrome. Nat Rev Dis Prim. 2018;5:18.

2. Bellani G, Laffey JG, Pham T, et al. Epidemiology, patterns of care, and mortality for patients with acute respiratory distress syndrome in intensive care units in 50 countries. JAMA J Am Med Assoc. 2016;315:788-800.

3. Zaccardelli DS, Pattishall EN. Clinical diagnostic criteria of the adult respiratory distress syndrome in the intensive care unit. Crit Care Med. 1996;24:247-51.

4. Villar J, Blanco J, Añón JM, et al. The ALIEN study: incidence and outcome of acute respiratory distress syndrome in the era of lung protective ventilation. Intensive Care Med. 2011;37:1932-41.

5. Sakr Y, Vincent $J L$, Reinhart K, et al. High tidal volume and positive fluid balance are associated with worse outcome in acute lung injury. Chest. 2005;128:3098-108.

6. Bein T, Weber-Carstens S, Apfelbacher C. Long-term outcome after the acute respiratory distress syndrome: Different from general critical illness? Curr Opin Crit Care. 2018;24:35-40.

7. Herridge MS, Tansey CM, Matté A, et al. Functional disability 5 years after acute respiratory distress syndrome. N Engl J Med. 2011;364:1293-304.

8. Neff TA, Stocker R, Frey HR, Stein S, Russi EW. Long-term assessment of lung function in survivors of severe ARDS. Chest. 2003;123:845-53.

9. Aisiku IP, Yamal JM, Doshi P, Rubin ML, Benoit JS, Hannay J, Tilley BC, Gopinath S, Robertson CS. The incidence of ARDS and associated mortality in severe TBI using the Berlin definition. J Trauma Acute Care Surg. 2016;80:308-12.

10. Kahn JM, Caldwell EC, Deem S, Newell DW, Heckbert SR, Rubenfeld GD. Acute lung injury in patients with subarachnoid hemorrhage: incidence, risk factors, and outcome. Crit Care Med. 2006;34:196-202.

11. Prabhakar H, Mahajan C, editors. Brain and Lung Crosstalk. New Delhi: Springer; 2020.

12. Gonzalvo R, Martí-Sistac O, Blanch L, López-Aguilar J. Bench-to-bedside review: brain-lung interaction in the critically ill: a pending issue revisited. Crit Care. 2007;11:1-5.

13. Tracey KJ. Physiology and immunology of the cholinergic antiinflammatory pathway. J Clin Invest. 2007;117:289-96.

14. Oddo M, Citerio G. ARDS in the brain-injured patient: what's different? Intensive Care Med. 2016;42:790-3.

15. Arksey H, O'Malley L. Scoping studies: towards a methodological framework. Int J Soc Res Methodol Theory Pract. 2005;8:19-32.

16. Liberati A, Altman DG, Tetzlaff J, et al. The PRISMA statement for re-porting systematic reviews and meta-analyses of studies that evaluate healthcare interventions: explanation and elaboration. BMJ. 2009;339:b2700.

17. Ranieri VM, Rubenfeld GD, Thompson BT, et al. Acute respiratory distress syndrome: the Berlin definition. JAMA J Am Med Assoc. 2012;307:2526-33.

18. Bernard GR, Artigas A, Brigham KL, Carlet J, Falke K, Hudson L, Lamy M, Legall JR, Morris A, Spragg R. The American-European consensus conference on ARDS. Definitions, mechanisms, relevant outcomes, and clinical trial coordination. Am J Respir Crit Care Med 1994;149:818-24.

19. Migdady I, Rice C, Deshpande A, et al. Brain injury and neurologic outcome in patients undergoing extracorporeal cardiopulmonary resuscitation: a systematic review and meta-analysis. Crit Care Med. 2020;48:e845.

20. Higgins JP, Altman DG, Gøtzsche PC, et al. Cochrane bias methods group; cochrane statistical methods group: the cochrane collaboration's tool for assessing risk of bias in randomised trials. BMJ 2011;343:d5928.

21. Wells GA, Shea B, O'Connell D, et al. The Newcastle-Ottawa Scale (NOS) for assessing the quality of nonrandomised studies in meta- analyses. Ottawa, ON, Canada, Ottawa Hospital Research Institute, 2012. (http:// www.ohri.ca/programs/clinical_epidemiology/oxford.asp.)

22. Murad MH, Sultan S, Haffar S, Bazerbachi F. Methodological quality and synthesis of case series and case reports. BMJ Evid Based Med. 2018;23:60-3. 
23. Bickenbach J, Zoremba N, Fries M, et al. Low tidal volume ventilation in a porcine model of acute lung injury improves cerebral tissue oxygenation. Anesth Analg. 2009;109:847-55.

24. Bickenbach J, Biener I, Czaplik M, et al. Neurological outcome after experimental lung injury. Respir Physiol Neurobiol. 2011;179:174-80.

25. Heuer JF, Pelosi P, Hermann P, et al. Acute effects of intracranial hypertension and ARDS on pulmonary and neuronal damage: a randomized experimental study in pigs. Intensive Care Med. 2011;37:1182-91.

26. Kamuf J, Garcia-Bardon A, Ziebart A, et al. Lung injury does not aggravate mechanical ventilation-induced early cerebral inflammation or apoptosis in an animal model. PLoS ONE. 2018;13:1-11.

27. Rodríguez-González R, Ramos-Nuez Á, Martín-Barrasa JL, et al. Endotoxininduced lung alveolar cell injury causes brain cell damage. Exp Biol Med. 2015;240:135-42.

28. Kamuf J, Andreas GB, Ziebart A, et al. Rosuvastatin influences cerebral inflammation and oxidative stress in experimental ARDS in pigs. Am Soc Anesthesiol 2017: The Anesthesiology Annual Meeting; Oct 21-25 2017; Boston, MA.

29. Fries M, Bickenbach J, Henzler D, et al. S-100 protein and neurohistopathologic changes in a porcine model of acute lung injury. Anesthesiology. 2005;102:761-7.

30. Klein $\mathrm{KU}$, Hartmann EK, Boehme $\mathrm{S}$, et al. $\mathrm{PaO}_{2}$ oscillations caused by cyclic alveolar recruitment can be monitored in pig buccal mucosa microcirculation. Acta Anaesthesiol Scand. 2013:57:320-5.

31. Kreyer $\mathrm{S}$, Muders $\mathrm{T}$, Luepschen $\mathrm{H}$, et al. The effect of pumpless extracorporeal $\mathrm{CO}_{2}$ removal on regional perfusion of the brain in experimental acute lung injury. J Neurosurg Anesthesiol. 2013;25:324-9.

32. Zampieri FG, Almeida JR, Schettino GP, Park M, Machado FS, Azevedo LCP. Factors associated with variation in intracranial pressure in a model of intra-abdominal hypertension with acute lung injury. Rev Bras Ter Intensiva. 2011;23:164-9.

33. Janz DR, Abel TW, Jackson JC, Gunther ML, Heckers S, Ely EW. Brain autopsy findings in intensive care unit patients previously suffering from delirium: a pilot study. J Crit Care 2010;25:538.e7-538.12.

34. Kim JS, Kim YJ, Kim M, et al. The impact of severity of acute respiratory distress syndrome following cardiac arrest on neurologic outcomes. Ther Hypothermia Temp Manag 2020. Epub 2020 May 22.

35. Hopkins RO, Gale SD, Weaver LK. Brain atrophy and cognitive impairment in survivors of acute respiratory distress syndrome. Brain Inj. 2006;20:263-71.

36. Hopkins RO, Weaver LK, Pope D, Orme JF, Bigler ED, Larson-Lohr V. Neuropsychological sequelae and impaired health status in survivors of severe acute respiratory distress syndrome. Am J Respir Crit Care Med. 1999;160:50-6.

37. Mikkelsen ME, Christie JD, Lanken PN, et al. The adult respiratory distress syndrome cognitive outcomes study: long-term neuropsychological function in survivors of acute lung injury. Am J Respir Crit Care Med. 2012:185:1307-15.

38. Hopkins RO, Suchyta MR, Snow GL, Jephson Al, Weaver LK, Orme JF. Blood glucose dysregulation and cognitive outcome in ARDS survivors. Brain Inj. 2010;24:1478-84

39. Bilotta F, Giordano G, Sergi PG, Pugliese F. Harmful effects of mechanical ventilation on neurocognitive functions. Crit Care. 2019;23:1-3.

40. Lahiri S, Regis GC, Koronyo Y, et al. Acute neuropathological consequences of short-term mechanical ventilation in wild-type and Alzheimer's disease mice. Crit Care. 2019;23:1-11.

41. Aktas O, Ullrich O, Infante-Duarte C, Nitsch R, Zipp F. Neuronal damage in brain inflammation. Arch Neurol. 2007;64:185-9.

42. Parnet P, Kelley KW, Bluthé RM, Dantzer R. Expression and regulation of interleukin-1 receptors in the brain. Role in cytokines-induced sickness behavior. J Neuroimmunol 2002;125:5-14.

43. Mascia L. Ventilatory setting in severe brain injured patients: does it really matter? Intensive Care Med. 2006;32:1925-7.

44. Picetti E, Pelosi P, Taccone FS, et al. VENTILatOry strategies in patients with severe traumatic brain injury: the VENTILO survey of the European Society of Intensive Care Medicine (ESICM). Crit Care. 2020;24:1-9.

45. Chikhani M, Das A, Haque M, Wang W, Bates DG, Hardman JG. High PEEP in acute respiratory distress syndrome: quantitative evaluation between improved arterial oxygenation and decreased oxygen delivery. $\mathrm{Br} J$ Anaesth. 2016;117:650-8.

46. Georgiadis D, Schwarz S, Baumgartner RW, Veltkamp R, Schwab S. Influence of positive end-expiratory pressure on intracranial pressure and cerebral perfusion pressure in patients with acute stroke. Stroke. 2001;32:2088-92.
47. Huseby JS, Luce JM, Cary JM, Pavlin EG, Butler J. Effects of positive endexpiratory pressure on intracranial pressure in mechanically ventilated dogs under hyperbaric oxygenation. J Neurosurg. 1981;55:704-5.

48. Kim J, Kim Y-J, Kim M, et al. Impact of lung compliance on neurological outcome in patients with acute respiratory distress syndrome following out-of-hospital cardiac arrest. J Clin Med. 2020;9:527.

49. Breit $H$, Jhaveri M, John S. Concomitant delayed posthypoxic leukoencephalopathy and critical illness microbleeds. Neurol Clin Pract. 2018;8:e31-3.

50. De Stefano P, Nencha U, De Stefano L, Megevand P, Seeck M. Focal EEG changes indicating critical illness associated cerebral microbleeds in a Covid-19 patient. Clin Neurophysiol Pract. 2020;5:125-9.

51. Fanou EM, Coutinho JM, Shannon P, et al. Critical illness-associated cerebral microbleeds. Stroke. 2017;48:1085-7.

52. Chen $\mathrm{H}, \mathrm{Xu} \mathrm{M}$, Yang $\mathrm{YL}$, et al. Effects of increased positive end-expiratory pressure on intracranial pressure in acute respiratory distress syndrome: a protocol of a prospective physiological study. BMJ Open. 2016;6:1-5.

53. Meduri GU, Annane D, Chrousos GP, Marik PE, Sinclair SE. Activation and regulation of systemic inflammation in ARDS: rationale for prolonged glucocorticoid therapy. Chest. 2009;136:1631-43.

54. Burad J, Bhakta P, George J, Kiruchennan S. Development of acute ischemic stroke in a patient with acute respiratory distress syndrome (ARDS) resulting from H1N1 pneumonia. Acta Anaesthesiol Taiwanica. 2012;50:41-5.

55. Kreisman NR, Soliman S, Gozal D. Regional differences in hypoxic depolarization and swelling in hippocampal slices. J Neurophysiol. 2000;83:1031-8.

56. Bartsch T, Döhring J, Reuter $\mathrm{S}$, et al. Selective neuronal vulnerability of human hippocampal CA1 neurons: lesion evolution, temporal course, and pattern of hippocampal damage in diffusion-weighted MR imaging. J Cereb Blood Flow Metab. 2015;35:1836-45.

57. Beilharz EJ, Williams CE, Dragunow M, Sirimanne ES, Gluckman PD. Mechanisms of delayed cell death following hypoxic-ischemic injury in the immature rat: evidence for apoptosis during selective neuronal loss. Mol Brain Res. 1995;29:1-14.

58. Sebat CM, Albertson TE, Morrissey BM. Cerebral gas embolism in a case of Influenza A-associated acute respiratory distress syndrome treated with high-frequency oscillatory ventilation. Ann Thorac Med. 2013;8:124-6.

59. Kahle KT, Walcott BP, Nahed BV, et al. Cerebral edema and a transtentorial brain herniation syndrome associated with pandemic swine influenza $A$ (H1N1) virus infection. J Clin Neurosci. 2011;18:1245-8.

60. Scully ER, Mark JE, McNeely FW, Ebeling SH, Philips LD. Case records of the Massachusetts General Hospital. Weekly clinicopathological exercises. Case 32-1997. A 43-year-old woman with rapidly changing pulmonary infiltrates and markedly increased intracranial pressure. N Engl J Med 1997;337:1149-1156.

61. Gale SD, Hopkins RO. Effects of hypoxia on the rain: neuroimaging and neuropsychological findings following carbon monoxide poisoning and obstructive sleep apnea. J Int Neuropsychol Soc. 2004;10:60-71.

62. Esser RW, Stoeckel MC, Kirsten A, et al. Structural brain changes in patients with COPD. Chest. 2016;149:426-34.

63. Horstmann A, Frisch S, Jentzsch RT, Müller K, Villringer A, Schroeter ML. Resuscitating the heart but losing the brain: brain atrophy in the aftermath of cardiac arrest. Neurology. 2010;74:306-12.

64. Hopkins RO, Weaver LK, Chan KJ, Orme JF. Quality of life, emotional, and cognitive function following acute respiratory distress syndrome. J Int Neuropsychol Soc. 2004;10:1005-17.

65. Hopkins RO, Weaver LK, Collingridge D, Parkinson RB, Chan KJ, Orme JF. Twoyear cognitive, emotional, and quality-of-life outcomes in acute respiratory distress syndrome. Am J Respir Crit Care Med 2005;171:340-347.

66. Larson MJ, Weaver LK, Hopkins RO. Cognitive sequelae in acute respiratory distress syndrome patients with and without recall of the intensive care unit. J Int Neuropsychol Soc. 2007;13:595-605.

67. Jackson JC, Hart RP, Gordon SM, Shintani A, Truman B, May L, Ely EW. Sixmonth neuropsychological outcome of medical intensive care unit patients. Crit Care Med. 2003;31:1226-34.

68. Fröhlich S, Murphy N, Doolan A, Ryan O, Boylan J. Acute respiratory distress syndrome: underrecognition by clinicians. J Crit Care. 2013;28:663-8.

69. Bassi TG, Rohrs EC, Reynolds SC. Systematic review of cognitive impairment and brain insult after mechanical ventilation. Crit Care. 2021;25:99. 